\title{
A Review on Recent Patents in Digital Processing for Cardiac Electric Signals (I): From Basic Systems to Arrhythmia Analysis
}

\author{
Rebeca Goya-Esteban, Óscar Barquero-Pérez, Felipe Alonso-Atienza, Estrella Everss, Jesús \\ Requena-Carrión, Arcadi García-Alberola and José L. Rojo-Álvarez**
}

\begin{abstract}
Department of Signal Theory and Communications, University Rey Juan Carlos, Madrid, Spain, Arrhythmia Unit, University Hospital Virgen de la Arrixaca, Murcia, Spain
\end{abstract}

Received: December 24, 2008; Accepted: January 19, 2009; Revised: January 20, 2009

\begin{abstract}
Cardiac electric signals are currently the most informative source about the heart rhythm and its disorders, and hence, the use of adequate digital signal processing techniques is necessary to yield reliable diagnostic parameters, either to the clinician or to automatic monitoring systems. A number of systems have been patented during the last years, which are grouped in this review according to their application scope. In this first part, techniques for electrocardiogram and intracardiac electrogram filtering, and for feature extraction, are first examined, then patents on arrhythmia analysis are then summarized. The wide number of basic systems for cardiac signal processing analysis that have been disclosed indicates that this field represents a main scenario in the near and middle future of cardiac health.
\end{abstract}

Keywords: Electrocardiogram, electrogram, digital signal processing, cardiac signal, arrhythmia, implantable devices, electrophysiology.

\section{INTRODUCTION}

Cardiovascular disease is the first cause of death in occidental countries [1]. The complex relationships among different processes in the heart are usually analyzed by means of cardiac images obtained, for instance, using magnetic resonance or ultrasounds, and by digital processing of cardiac signals. Mechanical signals (e.g., pressure in the vessels, blood flow) may be obtained invasively by catheterbased procedures or non-invasively and often they can be complemented with currently available image techniques. However, cardiac electric signals, which represent the controlling system of the heart, can only be obtained by measuring the voltage difference between two electrodes. This is a crude representation for a phenomenon which involves complex propagation patterns of three dimensional bioelectric currents moving through the asymmetric three dimensional cardiac structures. Nevertheless, most of the electrocardiography and cardiac electrophysiology knowledge has its basement on the analysis of cardiac electric signals, and a wide variety of systems to support their analysis have been proposed during the XX and XXI centuries.

Given that there is no current method for obtaining realtime medical images of electric cardiac activity in the clinical setting, we are attending today to the movement from atomic analysis of signals, with highly simplified methods, to modern systems, which aim to visualize the activity of the cardiac patterns in the heart. Hence, the role of signal processing techniques is becoming crucial when trying to build three-dimensional time-varying images of the heart

*Address correspondence to this author at the B004, Universidad Rey Juan Carlos, Camino del Molino s/n, 28943-Fuenlabrada (Madrid), Spain; Tel: (+34) 91488 8464; Fax: (+34) 9148875 00; E-mail: joseluis.rojo@urjc.es from electric signals. Also, digital signal processing has a central role in long-term monitoring applications, and techniques for this purpose continue to be disclosed $[2,3]$.

In this paper and in the companion one [4], we make a review on recent patents related to the application of digital signal processing techniques to cardiac electric signals. In this setting, it is worth to look not only to systems which are currently used in clinical practice, but also to disclosed systems which could be used in the near future.

The structure of the review is as follows. This first paper includes patents that are more closely related to basic digital signal processing algorithms for feature extraction in cardiac monitoring systems, as well as systems disclosed for applications related with cardiac arrhythmias. The companion paper [4] pays attention to the widely explored field of sudden cardiac death prediction, to the most advanced signal processing algorithm disclosed in this setting, and to the systems that have been proposed aiming to solve the inverse problem in electrocardiography.

In Section 2, a brief description of the nature of cardiac signals is given, including the components of the surface electrocardiogram (ECG) signals. Section 3 describes the methodology that has been used for selecting the related patents. Section 4 is devoted to patented algorithms for analyzing the ECG signals using techniques for extraction of simple yet representative features, as well as filtering and denoising. Section 5 deals with patented algorithms for intracardiac electrogram (EGM) analysis, including signals obtained from electrophysiological study (EPS) and from implantable cardiac devices (ICD). Section 5 contains patents on signal processing algorithms dealing with cardiac arrhythmias. Finally, Section 6 contains some conclusions on the specific topics in this part of the review. 


\section{CARDIAC SIGNALS}

The ECG is a graphic tracing that represents the electrical activity of the heart over time. A typical ECG tracing of a normal cardiac cycle (sinus rhythm) consists of a $\mathrm{P}$ wave, a QRS complex (consisting on the set of possibly present Q, R, and $\mathrm{S}$ waves), and a $\mathrm{T}$ wave see Fig. (1). Each wave represents a different stage of a heart beat, and their morphology and timing convey information that can be used for diagnosing those disorders that produce disturbances of the heart electrical activity. Atrial and ventricular depolarizations are given by the $\mathrm{P}$ wave and the QRS complex, respectively, whereas ventricular repolarizatition is given by the $\mathrm{T}$ wave. Atrial repolarization cannot usually be discerned from the ECG, since it coincides with the much larger QRS complex.

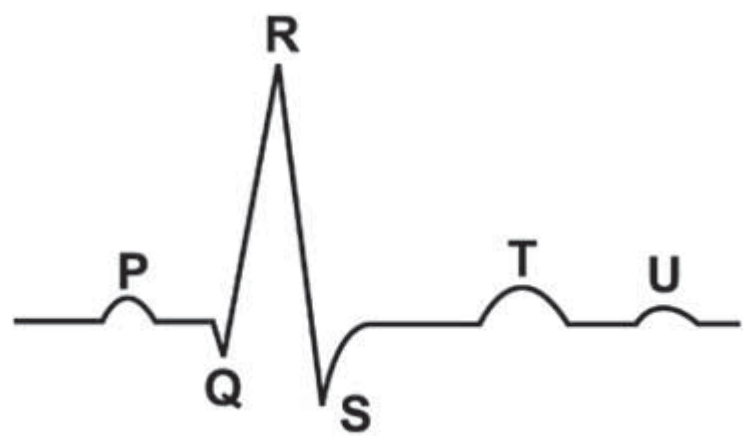

Fig. (1). Example of ECG during a cycle of sinus rhythm and most representative waves.

The electrical activity of the heart can be measured on the body surface by attaching a set of electrodes to the skin. The electrodes are positioned so that the spatiotemporal variations of the cardiac electrical field are recorded. The difference in voltage between a pair of electrodes is referred to as a lead, and the ECG is typically recorded with a multiple lead configuration. The electrode wires are connected to a differential amplifier, which has to be specially designed for bioelectrical signals. Specifically, an amplifier with high gain and large dynamic range is required, since the ECG amplitude ranges from some few $\mu V$ to about $10 \mathrm{mV}$. The ECG is usually obtained by a standard 12-lead arrangement, but it can be obtained by using other lead systems, for instance, the orthogonal XYZ lead configuration. After the ECG signal acquisition, the resulting signal must be processed and usual stages consist of filtering for noise reduction, QRS detection, wave delineation and data compression.

Equipments that contain ECG acquisition systems can be found, for instance, in intensive care units and in cardiac electrophysiology and arrhythmia units, for continuously monitoring the state of the heart rhythm of a patient under surveillance or cardiologic interventions. Also, ambulatory monitoring (Holter systems) consists of the recording of the ECG usually from two or three leads (up to 7 leads in some commercial systems) during long periods ( 24 hours or more) and its subsequent analysis, for a more detailed diagnosis of the patient's cardiac state. A number of ECG signal analysis systems have been proposed to process signals recorded under particular conditions. Common to all these systems is a set of algorithms which condition the signal with respect to different types of noise, extract basic ECG measurements, and compress the data for efficient storage or transmission [2].

\section{SEARCH METHODOLOGY}

Our initial search of patents was made by using the ISI Web of Knowledge engine (www.isiwebofknowledge.com), which is an online academic database from Thomsom Scientific. Free access was available to our institution by Spanish Ministry of Science and Innovation (MICINN) and by Spanish Society for Science and Technology (FECYT) through www.accesowok.fecyt.es.

The patent search procedure was made by using Derwent Innovations Index $^{\circledR}$ database, among the possible ones available from ISI Web of Knowledge in its search procedures. For search simplification, nine different groups were considered, aiming to group into related sets the patents with specific keywords. Table 1 gives some detail on the search parameters and on the number of retained patents.

After this search, all the patents were examined and included according to the patent abstract containing information on the use of digital signal processing algorithms in cardiac electric signals within the disclosed system. Hence, only the full text of those patents possibly related with digital signal processing and electric signals were examined with full detail. Patents that were duplicated, or which did not represent any disclosure of signal processing algorithms for cardiac signal analysis, were eliminated after this stage. All the electronic documents containing patents were downloaded from Europe's Newtwork of Patent Databases (www. espacenet.com), which is a free service for patent search and download, developed by European Patent Office (EPO) together with the member states of the European Patent Organisation.

\section{ECG FEATURES}

Many systems have been patented for extraction of the ECG relevant features, given the importance of this stage in the subsequent signal analysis processes. Following, some of the most important patents in this field are presented according to their orientation in this field.

\subsection{Lead Systems}

A standard 12-lead ECG is normally acquired by using ten electrodes placed at specific locations on the patient's body. It has been pointed out that it is possible to reduce the number of leads to be used, due to the redundancy in the cardiac information present in each of the standard ECG leads.

In [5], a method is presented for creating an ECG virtual lead. ECG signals from at least two leads are generally used in order to generate a vectorcardiogram (VCG) representation of the cardiac electrical activity. The method includes steps of obtaining a first ECG signal from a first lead having a first angle and a second ECG signal from a second lead having a second angle. Then, the two ECG signals are transformed into a VCG, likewise the VCG is transformed 
Table 1. Parameters Used in the Search Procedure and Number of the Retained Patents for Each Search

\begin{tabular}{|c|c|c|c|}
\hline Topic & Timespan & Refined By & Number of Patents \\
\hline (ECG) OR (electrogram) AND (signal) & $2000-2008$. & $\begin{array}{l}\text { Topic }=(\text { cardiac) } \text { AND Subjectareas }= \\
\text { (engineering or general \& internal medicine or instruments } \\
\text { \&instrumentation or computer science). }\end{array}$ & 695 \\
\hline $\begin{array}{c}\text { (Cardiac OR ecg or egm) AND (algorithm) } \\
\text { AND (signal processing) }\end{array}$ & All years & & 48 \\
\hline (Arrhythmia) AND (signal processing) & All years. & & 71 \\
\hline (Arrhythmia AND (signal processing) & All years & & 71 \\
\hline (Fractal OR chaos OR entropy) & All years & & 40 \\
\hline (Heart rate turbulence) & All years & Topic $=($ processing $)$ & 3 \\
\hline ("Late potentials") & All years & & 17 \\
\hline (T wave alternans) & All years & & 15 \\
\hline (Non-contact mapping) & 1998-2008 & & 26 \\
\hline (Heart rate variability) & 1998-2008 & Topic $=($ signal processing $)$ & 23 \\
\hline
\end{tabular}

into a virtual ECG signal representing a virtual lead. The virtual ECG signal can be used to replace an ECG signal from a lead that has been lost or corrupted. Also, virtual ECG signals can be estimated at angles for which there are no recording electrodes on the patient.

A method is disclosed in [6] for analyzing lead-limited ECG signals. Analysis of one, two, or three lead ECG is based on computing a limited number of ECG signals, originating from two or three electrodes. The main benefit of using limited lead ECG is its simplicity, but its analysis is difficult because they provide limited amount of information in comparison to 12-lead ECG. The invention comprises a step of receiving an ECG signal representative of heart activity from a plurality of ECG leads, a step of pre-processing the ECG signal and a step of identifying heart arrhythmias by means of expert systems (artificial neural network, ANN) and statistical decision methods. Verification of detected heart arrhythmias is also considered. The method further comprises a step of detecting auxiliary data, such as averaged heart rate, rhythm irregularities, ST segment elevation, AC interference, or noise level. In [7], an apparatus is presented for extraction of electrocardiographic signals using less than ten electrodes. The source of each of the collected electrocardiographic signals is identified as to the locations on the patient's body to which the electrodes are placed and a twelve lead ECG is derived from the collected electrocardiographic signals, mathematically deriving the ECG leads that are not directly measured. Then, an interpretation algorithm is applied to the measurements, which is derived by using a combination of a non-linear regression model (ANN) and a rule induction model using a binary decision tree and entropy gain.

\subsection{ECG Filtering}

Wide attention has been paid to the design of filters, either from linear or nonlinear structures, for the removal of baseline wander and powerline interference. Removal of muscle noise represents another important filtering problem, which is more difficult to handle because of its substantial spectral overlap with the ECG [2].

A method for reducing noise in the ECG is included in [8]. The method acquires the ECG, compares it to a representative signal (generated from previous beats or from related biological signals), and generates a predicted signal when the ECG signal sufficiently matches the representative signal. The predicted signal is subtracted from the ECG signal to produce a second signal which is filtered. The predicted signal and the filtered signal are then combined to produce a noise-reduced signal.

In [9], a low pass filter specially adapted for its use with ECG equipment is designed. Several low-pass finite impulse response (FIR) filter stages are connected in tandem, each of them having a distinct cut-off frequency. Filter stages can be set to on or off, but the time delay is always constant. Each stage includes a time delay buffer that has an input for receiving and storing digital data for predetermined time intervals, the derivation from the buffer output of an average-over-time noise signal component falling within a predetermined frequency band and the selective subtraction of the noise component.

The ECG can be distorted in patients with implanted pacemakers due to the delivery of electric pulses from the device. In [10], a signal processor is presented, which digitally filters the signal from the body, resulting in an ECG 
signal and a signal that identifies the presence and timing of signals from a pacemaker in the body. The device comprises the following stages: An anti-aliasing analog filter, that receives an analog signal from the body and generates a filtered analog signal, and an analog-to-digital converter; A first digital filter, which receives a signal from the body and generates a pacemaker signal as a function of pacing pulses supplied by a pacemaker; A second digital filter, which receives the digital signal and generates an ECG signal; And a third digital filter, which receives the digital signal and generates a signal indicative of the quality of the electrical connection of an electrode to the body.

In [11], a method is disclosed to determine an axis shift in an ECG waveform and to use this for filtering out bad ECG information. Also, an adaptive filter can be used to determine physiologic conditions even after an axis shift.

The presence of a QRS complex and its occurrence time is fundamental in most of ECG signal processing systems. The design of a QRS detector is a necessary condition, since poor detection performance may propagate to subsequent processing steps, hence limiting the overall performance of the system [2]. The RR series consists of a plurality of samples $(R R i)$ defining respectively the time intervals between two successive heart beats. In [12], a method for filtering RR series obtained after sampling an analog cardiac signal is shown, consisting in detecting and automatically filtering the erroneous samples in the RR series. To detect whether an $R R i$ sample is erroneous, its value is compared with a self-adaptive threshold, which is calculated from the mean and the standard deviation of the $\mathrm{N}$ samples in a sliding window.

\section{3. $P$ Wave Detection}

In a normal heart, the atrial electrical activation begins in a region called the sinus node, where specialized cells activate themselves in an automatic, near periodic way. In some atrial arrhythmias the origin of the electric impulse may shift from the sinus node to other areas, triggering an abnormally high rate and producing premature atrial contractions (PAC), either isolated or grouped. When three or more PAC occur in a row, the term atrial tachycardia is used. The $\mathrm{P}$ wave of such premature beats has a different morphology and it may overlap with the $T$ wave of the preceding beat, making its recognition difficult or even impossible.

In [13], a computerized method is disclosed for performing real time cardiac analysis through the processing of incoming electrical signals from the heart. The device displays a derived $\mathrm{P}$ wave without any overlap with a preceding $\mathrm{T}$ wave during a PAC and allows the practitioner to objectively compare derived $\mathrm{P}$ waves to determine if they are emanating from the same focus. The signal processor allows the user to mark a beginning and an ending point of the first ECG signal to define a waveform segment as a reference template. It acquires data from multiple leads and identifies and displays the best fit between the reference template and the acquired data, according to their correlation coefficient.
In [14], an algorithm to enhance the ability to visualize $P$ waves in surface ECG data is presented. The ECG data is selectively passed through two different processing channels. The first one includes a low pass filter with a cut-off frequency capable of attenuating spectral energies characteristic of ventricular depolarization while passing atrial depolarization. The second one comprises a signal delay configured to match the signal delay inherent in the low pass filter. The filtered data is inserted into the data stream in place of the raw input signal for a predetermined time interval, long enough to ensure the occurrence of an atrial depolarization signal, in order to detect an atrial event.

Another system for analyzing the $\mathrm{P}$ wave of an ECG signal is disclosed in [15]. The method acquires the ECG signal in the form of VCG, detects a wave from the ECG signal with a template and calculates the parameters of the $\mathrm{P}$ wave. Dynamic changes of $P$ wave are tracked by comparing every detected $\mathrm{P}$ wave to the reference $\mathrm{P}$ wave in a defined time period. The computed parameters are presented as points on a trend graph. The method is capable of detecting the atrial extrasystoles and separating them into subclasses according to their morphology.

\section{EGM ANALYSIS}

Different from the skin ECG, intracardiac EGM signals record the electrical activity of the heart from electrodes attached to catheters placed within the cardiac chambers. EGM signals stand for one of the principal information tools in the clinical EPS and in ICD.

\subsection{EGM from EPS}

The most reliable method currently available for identifying defects in the cardiac conduction system is a diagnostic procedure called EPS, which consists of inducing and studying the mechanism of some arrhythmias [16]. During this intervention, slender wires, called catheters, are introduced through the skin into a large vein (e.g. femoral or subclavian), and advanced with X-ray guidance into the heart chambers, for reaching places in the heart near the sinus node, the atrioventricular (AV) node, the His bundle or the ventricles. EPS catheters can work in two modes, either exploratory (sensing the electrical signal) or pacing (delivering controlled stimulation pulses), and they can be connected to a visualization system and to a pulse-generator device. The interior surface of the heart is then subjected to convenient electrical stimulation pulses protocols, in order to determine the underlying mechanism of the arrhythmia previously observed in the patient, and therapeutic procedures can be delivered, such as cardiac ablation with high-energy radiofrequency (RF) energy, in order to destroy the arrhythmia mechanism.

\section{Support to EPS Procedures}

A number of simple signal processing algorithms have been proposed for giving support to conventional EPS procedures. In [16], a system is disclosed for identifying at least one of the $\mathrm{A}, \mathrm{V}$ and $\mathrm{H}$ waves (at the atria, ventricle, and His bundle level, respectively) see Fig. (2), on at least one of the available EGM signals. In [17], an ablation system is disclosed which uses a catheter with a distal end and a 


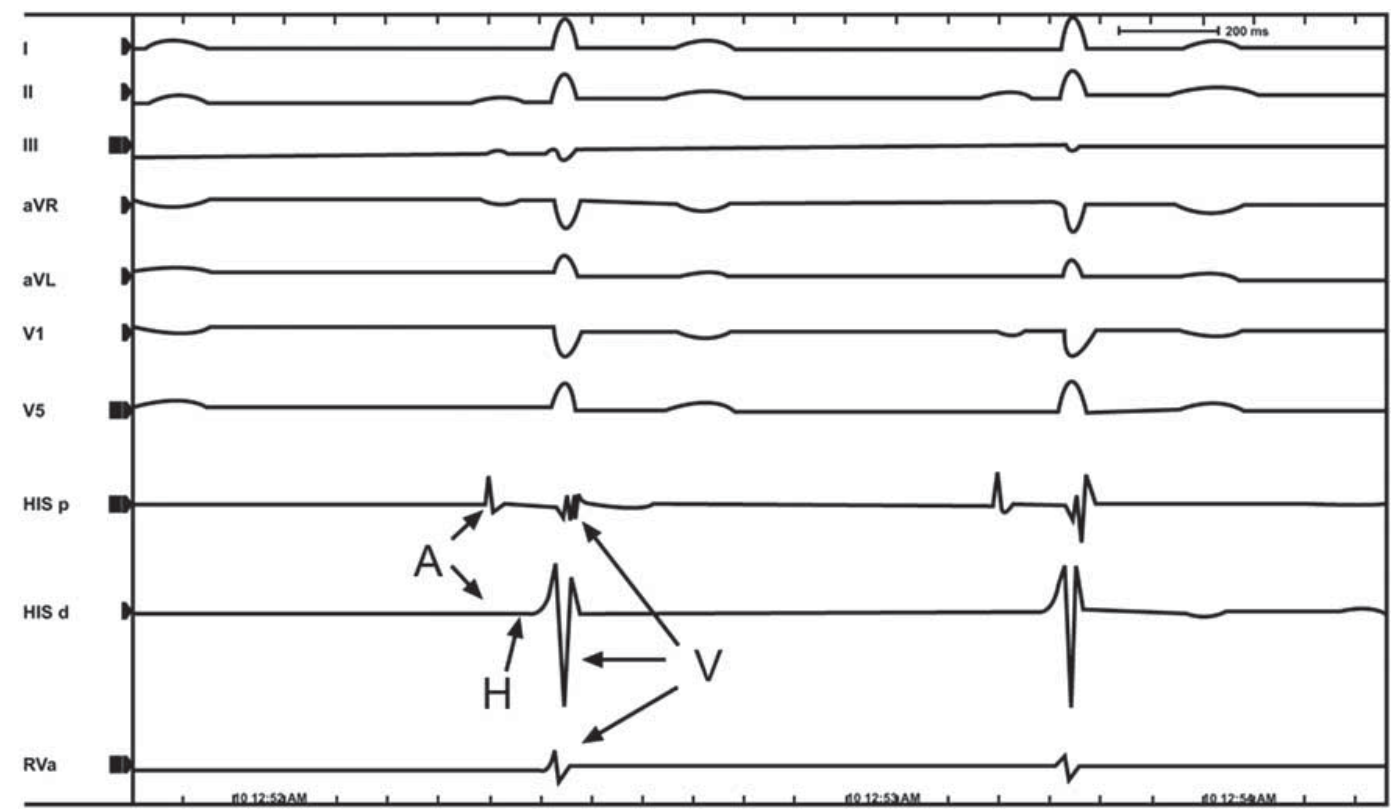

Fig. (2). Example of real EGM and ECG leads during an electrophysiological study. The first seven are ECG leads while the three last correspond to EGM leads. An atrial EGM (A), a ventricular EGM (V) and a His deflexion (H) are marked.

plurality of electrodes adjacent to it. A phase-locked all pass filter, connected between the catheter and the patient interface, suppresses the interference of the RF ablation on the EGM during the ablation process. Another ablation supporting system is introduced in [18] with a different working principle, namely, the use of sensed acoustical energy, in combination with the ECG signal, for detection of instability of ablation electrode and crater formation during ablation. In [19], a system with signal generation capabilities delivers a paced input signal (pulses spaced by pacing intervals) to a driving region of the heart, receives and analyzes the output EGM from that region, and identifies the delay between input and output signals to determine predisposition to some cardiac conditions, such as sudden cardiac death.

\section{Multiple Signals and Mapping}

Advanced applications of digital signal processing in EPS include the analysis of multiple electrode signals and the support to cardiac mapping. In [20], a system is disclosed for analyzing cardiac arrhythmia using timing and variability of relationships between EGM features. The system simultaneously records a plurality of EGM, each from a region of the heart. Several fiducial markers, indicative of a feature of interest, are obtained on a selected EGM and multiple segments of each EGM are aligned with respect to the reference. The aligned segments of each EGM are averaged, and features can be obtained for analyzing their timing and variability. Focal and reentrant mechanisms are given as representative examples. A highly comprehensive system is proposed in [21], which analyzes ECG and EGM to identify and separate electrical activity corresponding to different but superimposed events in the heart see Fig. (3). It can select simple signal processing tools, such as the correlation coefficient, power spectrum or filtering, as well as complex analysis actions, such as signal conditioning, time-spatial correlation, timing analysis, or spatial magnitude comparison. The multifunction system is designed for surface ECG and intracardiac diagnosis and localization, catheter guidance, determination of ablation success, and arrhythmia prediction.

\subsection{EGM from ICD}

The basic functionality of ICD includes automatic cardiac event sensing, arrhythmia detection, and therapy administration. The most usual ICD are pacemakers, implantable cardioverter defibrillators and resinchronization pacemakers and defibrillators. Patents in this section are aimed at enhancing and extending ICD functions by applying techniques of signal analysis on EGM recordings from the device catheters.

\section{Sensing Capabilities}

Several patents have sought to improve ICD sensing capabilities. In [22], a system is disclosed which includes a monitor configured to detect an RF artefact from the ICD signals, together with circuitry for processing the artefact to eliminate a false heart beat identification. The system also identifies paced heart beats and detects the occurrences of pacing from the device that fails to capture the surrounding cardiac tissue. In [23], a method is devised that compares EGM waveforms recorded by true and integrated bipolar leads in order to determine which lead allows a more accurate sensing of cardiac events see Fig. (4). Similarly, a method is proposed in [24] for identifying and classifying oversensing based on the comparison of the occurrence of Rwaves in both bipolar and pseudo-monopolar leads. An atrial $\mathrm{EGM}$, i.e. from catheters in an atrial location, senses atrial events, but an undesired artefact of far field ventricular activity ( $\mathrm{V}$ wave) can be present in this kind of recordings. In [25], a morphological model of an estimated far field or template, is selected from a plurality of templates, according to the nature, rate, and timing of atrio-ventricular events and 


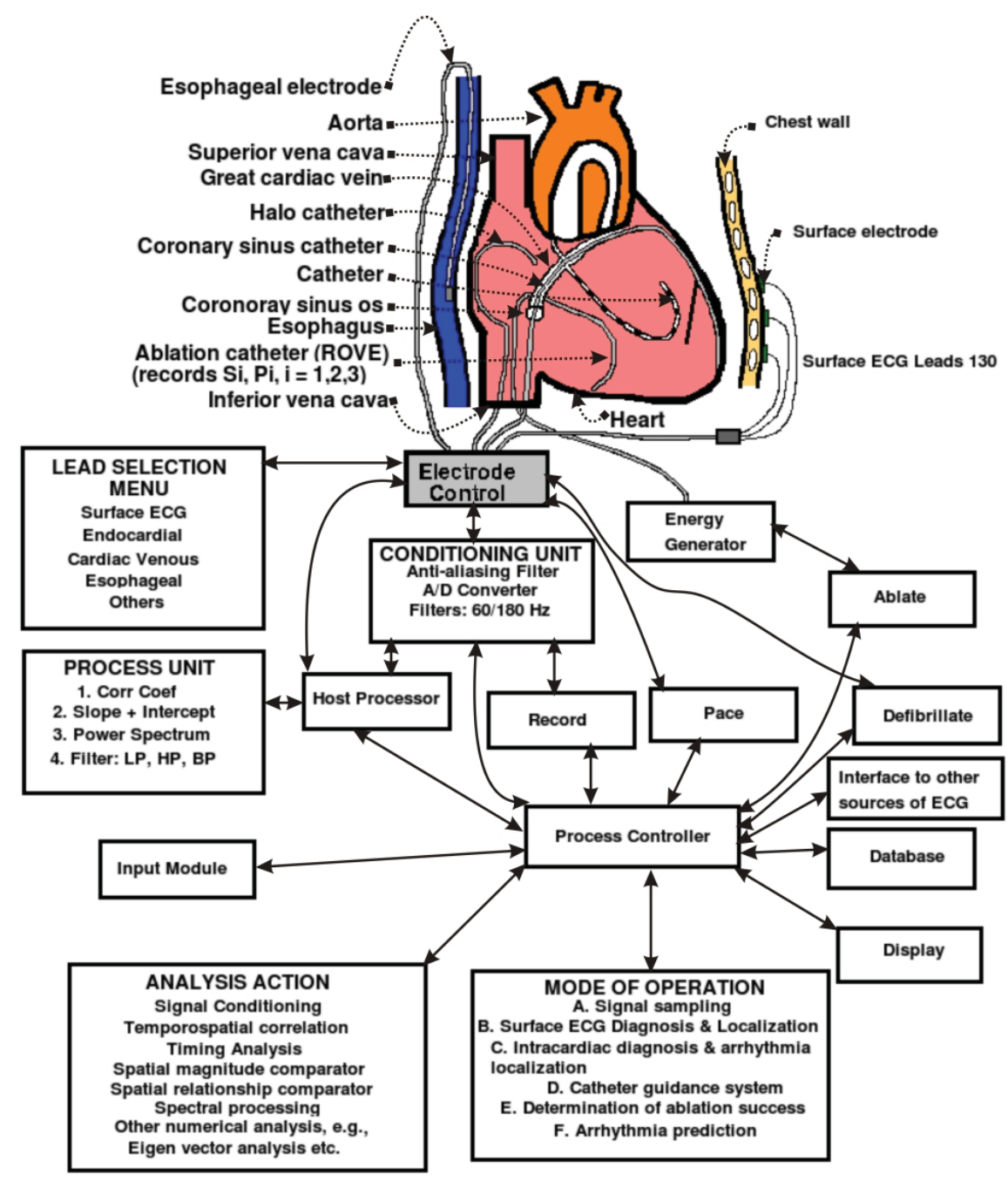

Fig. (3). Example of system structure for EPS supporting multisystem, including advanced signal processing of ECG and EGM (adapted from [19]).

the estimated far field $\mathrm{V}$ wave is then subtracted from the atrial EGM signal. In [26], measuring the modulation of the amplitude of an evoked response is disclosed in order to automatically adjust the detection threshold. In [27], inventors envision that the sensitivity of sensing leads could be adjusted in accordance with the magnitude of the noise floor, which is obtained from the density of local peaks in the EGM signal, from which a statistic, such as mean, median or mode, can be estimated.

\section{Event Detection}

Several inventions have focused on the development of improved cardiac event detectors. A detector based on morphology analysis is proposed in [28], consisting of a bank of filters with symmetric and antisymmetric impulse responses that are designed to detect biphasic and monophasic EGM waveforms. In [29], a system is presented including a wide QRS detection and alerting system. In [30] and [31], inventors envision that detection of acute myocardial infarction and/or ischemia would be based on the evaluation of ST deviation, which is defined as the difference between the average ST segment voltage and the average PQ segment voltage. Based on this, a detector is designed in which the ST shift, i.e. the difference between the ST deviation on a single beat and a baseline average ST deviation, is calculated and compared with a predetermined detection threshold.

Exemplary methods have also been developed to enhance detection and characterization of cardiac activity. In [32] and [33], authors use ensemble averaged EGM obtained under a plurality of conditions, such as native and evoked stimulation, for exemplary analysis. In [34], a method is devised to identify the timing of the His electrical activity by subtracting a ventricular EGM from an atrial EGM.

\section{Arrhythmia Detection}

The development of methods for arrhythmia detection and classification from EGM has been central in some recent patents. In [35], a detection scheme is proposed which estimates EGM measured parameters, such as heart rate, V wave width, V wave slew rate, amplitude and stability. A method is included in [36] for detecting arrhythmias based 


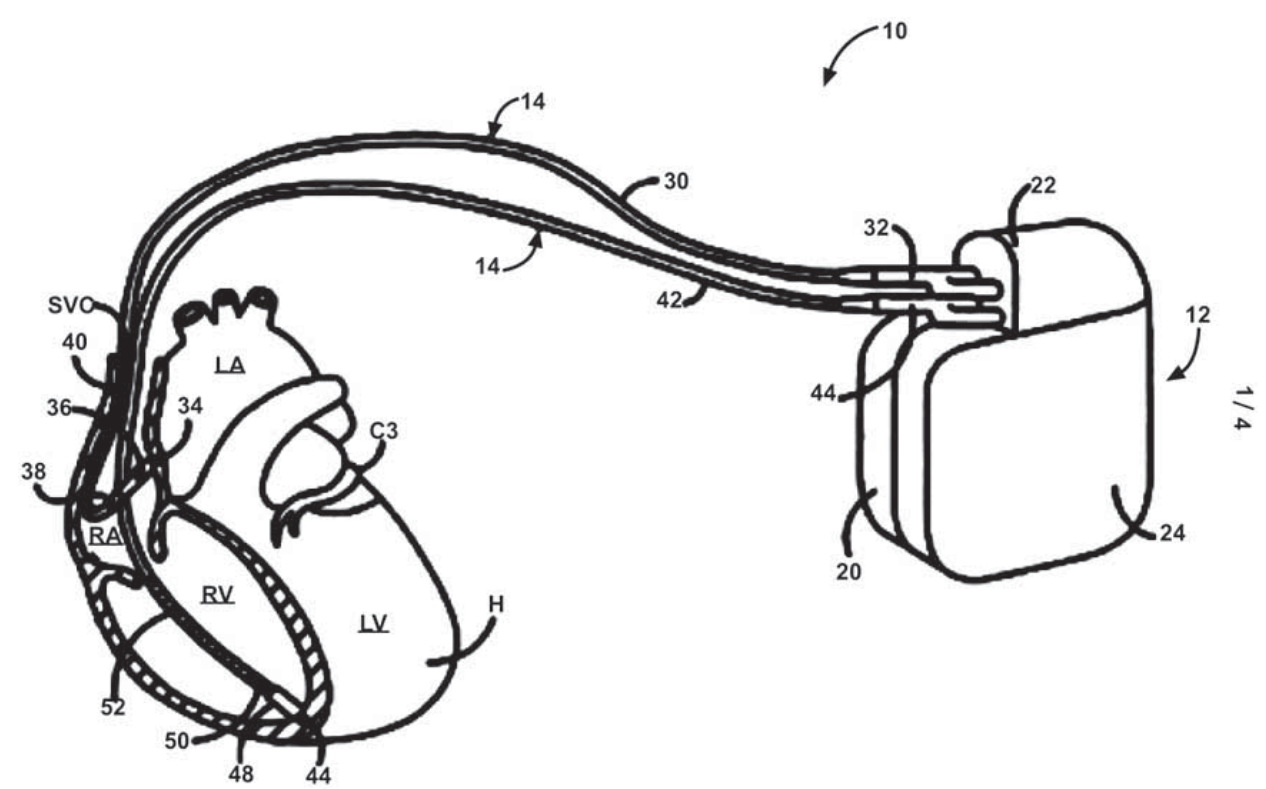

Fig. (4). Lead configuration and acquisition in an ICD (from [23]).

on the measure of $\mathrm{A}-\mathrm{A}, \mathrm{A}-\mathrm{V}, \mathrm{V}-\mathrm{A}$ or $\mathrm{V}-\mathrm{V}$ timing intervals, heart rate, onset characteristics of the intervals and EGM morphology. Another method is developed in [37,38] to discriminate between supraventricular and ventricular arrhythmias, which uses as a basis the morphology of the EGM waveform. Another method is proposed in [39] to discriminate between supraventricular and ventricular arrhythmias, which analyzes the timing of atrial and ventricular EGM following tachycardia recurrence subsequent to a train of simultaneously delivered anti-tachycardia pacing. In [40], researchers aim to improve arrhythmia diagnosis from EGM by using prior and conditional probabilities information, such as domain expert information, episode metrics and patient metrics. For support when analyzing large recordings of EGM, clustering methods are developed in [41] which group EGM waveforms according to morphological and chronological criteria.

\section{Other Improvements}

Inventors have also concentrated on enhancing ICD performance and functionality. Some methods have been developed for automatically setting ICD operating parameters. In $[42,43]$, methods for setting ICD pacing parameters are specified, such as the atrio-ventricular delay interval in $\mathrm{A}-\mathrm{V}$ sequential pacing modes, based on the measurement of intrinsic cardiac conduction times. A method is proposed in [44] for determining timing points for $\mathrm{T}$ wave shocks that compares two waveforms from a single EGM or from two different EGM. In [45], a method is designed for automatically adjusting the $\mathrm{V}-\mathrm{V}$ time interval in biventricular stimulation. This method consisted of an optimization algorithm that aims at minimizing the $\mathrm{V}$ wave recorded by far field leads.

\section{ARRHYTHMIAS}

Cardiac arrhythmias are due to alterations in the cardiac impulse origin or in its delivering to the heart. Bradyarrhy- thmias are due to a slowing in the heart rate and they are usually treated with drugs or pacemakers, whereas tachyarrhythmias are due to an increase of the heart rate and different therapies can be required according to their nature (shock or defibrillation, cardioversion, ablation, drugs). The most life-threatening arrhythmias are those with anatomical origin at the ventricular level, called ventricular tachycardias (VT), as they compromise the blood pumping to the body, whereas supraventricular tachycardias (SVT) (with anatomical origin at atrial or AV node level) are usually better tolerated.

\subsection{Ventricular Fibrillation}

Sudden cardiac arrest is a life-threatening condition, typically caused by ventricular fibrillation (VF), an arrhythmia in which cardiac impulse looses its rhythm and the heart is unable to pump the blood to the body. In order to restore the sinus rhythm, electrical defibrillation therapy has to be delivered, which consists of the application of a strong electric pulse to the heart, which can be done using an external or an implantable cardiac defibrillator.

In recent years, automated external defibrillators (AED) have been successfully used by emergency responders and they are now easily accessible in many public places, being suitable to be used by standers with minimal or no medical training [46]. When the patient is in VF, the shock has to be delivered in some few seconds before damage or death, whereas an inappropriately delivered shock, e.g. during sinus rhythm, can start a new VF episode. Therefore, fast and safe algorithms for VF detection are required which do not depend on the ability of the user for recognition of the rhythm characteristics in the ECG.

A number of patents have been proposed for improving AED performance, which usually focus either on advanced VF detection methods from the ECG or on the use of additional sources of signals to be combined with the ECG. 


\section{Advanced VF Detection Methods}

In [47], a rate-based algorithm for ECG analysis allows to discriminate between candidates either for unsynchronized shock (VF) or for cardioversion (VT). A more advanced algorithm is described in [48], which periodically measures the cardiac frequency from zero crossing of the ECG, heart beats and RR intervals during a period. A probability weighting is given according to a scale specific from each criterion and their summation yields an overall probability of arrhythmia during this period. A two stages VF detector from ECG analysis is proposed in [49], consisting of an adaptive nonlinear section (preferably an ANN with multiple output) followed by a linear section (preferably a sliding window of a given length, such as a tapped time-delay filter). The system is therefore capable of differentiating among several rhythm patterns. A method to exclude artefacts is also disclosed.

\section{Additional Signal Sources}

In addition to defibrillation, cardiopulmonary resuscitation (CPR) is often needed to treat cardiac arrest. CPR involves a periodic chest compression and artificial ventilation of the patient in order to maintain minimal but critical levels of circulatory and respiratory functions. Excessive patient motion can have a misleading effect on the AED algorithms for VF detection from the ECG. In [46, 50], AED are disclosed which are capable of determining if the patient is undergoing motion, in that case starting ECG analysis after an elapsed time (10 or $30 \mathrm{~s}$ ). Excessive motion is determined either from a sensor or from patient impedance (stroke volume of the heart) values and its fluctuations compared with upper and lower limits. The use of cardiographic impedance waveform for deciding whether to deliver therapy is more extensively developed in [51]. Cardiac output is estimated from the heart rate and the impedance, and further correlated to shockable and non-shockable conditions. Somehow detailed time scale processing and fuzzy logic analysis are also disclosed for fiducial point identification. In [52], the pulse signal is additionally sensed and analyzed in conjunction with the ECG signal to decide whether to deliver therapy. Periodicity analysis is proposed to check for coherence between both signals. In [53], an AED is disclosed capable of detecting a pediatric patient and then modifying the ECG analysis algorithm and the therapy level.

\subsection{Atrial Fibrillation}

Atrial Fibrillation (AF) is the irregular and rapid randomized activation of the atria due to a rapid succession of cardiac impulses, leading to atrial mechanical standstill and a fast and completely irregular ventricular rhythm, which is usually the cornerstone for the automatic detection or the arrhythmia. On the ECG, the $\mathrm{P}$ wave is replaced by fibrillation waves varying in shape, size and timing, with rates over 350 cycles per minute. AF is the most common arrhythmia in clinical practice, especially in the elderly. A number of patents have been proposed for AF detection, which can be roughly grouped into three orientations, namely, the analysis of QRS irregularity, the analysis of the atrial electrical activity after removal of QRS complexes and clinical monitoring.

\section{QRS Analysis}

In [54], a scheme is disclosed which uses the inter-beat variance above a threshold for classifying an ECG segment as AF. In [55], ratios of the current R-R intervals are first made to previous $\mathrm{R}-\mathrm{R}$ intervals and multiple moving averages are taken on the ratio results. Second, the ratios are compared to an upper and a lower threshold to be included in a second averaging. If the difference between both averages exceeds a threshold, AF is determined to be present. In [56], several (2 to 10) autorregressive coefficients of the RR series are obtained, and subsequently compared, by means of a test, to standard coefficients derived from patients not suffering from atrial fibrillation.

\section{Atrial Activity Analysis}

In [57], a method is disclosed for monitoring the progression of $\mathrm{AF}$ and to detect its susceptibility for termination. The processing algorithm cancels the QRST segment of the cardiac signal for excluding the ventricular electrical activity and then it uses the peak frequency of Fourier analysis and compares to a threshold for withholding anti-tachycardia delivery and to allow self termination of the episode.

\section{Monitoring}

In [58], a system is devoted to daily monitoring longterm $\mathrm{AF}$, estimating the likelihood of an arrhythmia from ECG detailed analysis and indicating the condition to the patient or to a physician for a review. In [59], a classification system is disclosed for predicting the postoperative risk of AF from a plurality of features and the subsequent calculation of an AF index.

\subsection{Ventricular Tachycardias}

Apart from VF and AF, also VT have been paid attention in recent patents. Algorithms for detecting the presence of VT in the ECG either propose the use of new ECG features or use combinations of established features.

\section{New ECG Features}

In [60], a composite QRS template is obtained during sinus rhythm, and derivative analysis is proposed for predicting ventricular tachycardia, according to several criteria. First, the last $40 \mathrm{~ms}$ are measured to obtain an indication of presence of high frequency Late Potentials (LP). Second, the QRS width is also used as an indicator of VT. Finally, the first or higher order derivatives of each beat are compared with the standard, by means of parameters such as the number of transitions and their time position, and area displaced by the pulse widths. A more sophisticated analysis is disclosed in [61], where curvature parameters for tachyarrhythmia detection and discrimination are disclosed. Each turn in the ECG is represented by a characteristic point in a curvature series, including time (centre of the turn), value (amplitude at time), area (direction and degree of the turn), and width (duration) parameters. The rhythm is decided after the analysis of the information in the curvature series. 


\section{Feature Combinations}

In [62], a system is disclosed including an ANN stage for arrhythmia discrimination. The system receives the ECG, extracts a set of features from a signal window, and compares them with a library of predefined examples by using distance measurements. A similar scheme is proposed in [63] for cardiac disease diagnosis, comprising not only tachycardia but also other disorders as ischemia.

\section{CURRENT \& FUTURE DEVELOPMENTS}

Digital signal processing constitutes nowadays an integral part of nearly every device in the biomedical industry. The benefits of digital approaches to signal processing are well known; among others, it is worth mentioning improved immunity to noise, more flexible processing schemes, more accurate results and easy implementation. Looking back, the development of digital signal processing techniques has contributed to the advancement of the biomedical industry providing with solutions to previous engineering challenges as much as with sources for innovating and creating new fields of research.

\section{ECG features}

The ECG features extraction is a capital field, since subsequent stages in signal processing depend on its quality. Many patents have focus on reducing the number of leads to be used on the ECG signal extraction in order to simplify and improve this stage. Also, much attention has been paid to the ECG filtering stage, specifically designed for reducing noise on the ECG signal in diverse situations, for instance, to correctly detect the presence of QRS complexes. The P-wave detection enhancement in the surface ECG has been also a deeply studied issue. The investigation and development in these areas is expected to continue due to its relevance.

\section{EGM Analysis}

Cardiac electrophysiology is a medical discipline whose methods and techniques have evolved hand in hand with those of biomedical engineering and it has also benefited from the development of digital signal processing techniques. The role of digital signal processing in cardiac electrophysiology manifests in the large amount of patents in which techniques of digital processing of electric cardiac signals are described. In this paper, we have reviewed some of the most relevant recent patents involving digital processing of electric cardiac signals. In some cases, the technique of digital signal processing was one among several aspects of a whole system that was the subject of the patent; in others, the technique of digital signal processing constituted the actual subject of the patent.

New applications of digital signal processing are expected to emerge in the future. One of the main challenges of current ICD is devising detection methods that discriminate between SVT and VT with a high specificity. The introduction of new ICD lead systems that allow for multiple sites recording has opened the possibility to obtain a more global picture of heart dynamics, from which detection algorithms can benefit. The interplay with other biomedical technologies, such as resonance image, computed tomography, and echocardiography, will be one of the main driving forces of digital signal processing in cardiac electrophysiology. The integration of medical images with multidimensional cardiac electric signals, which has already been put into practice commercially, will provide us with increasingly better estimations of heart dynamics. Therefore, we expect to witness a strong tendency towards developing methods of multidimensional electric recordings, which account for space-time patterns.

\section{ACKNOWLEDGEMENTS}

This work has been partially supported by Projects TEC2007-68096-C02/TCM from Ministerio de Ciencia e Innovación and URJC-CM-2008-CET-3732 from Comunidad de Madrid.

\section{CONFLICT OF INTERESTS}

None.

\section{REFERENCES}

[1] Zipes DP, Jalife J. Cardiac electrophysiology: From cell to bedside. WB saunders company, Phyladelphia, 1995.

[2] Sörnmo L. Laguna P. Bioelectrical signal processing in cardiac and neurological applications. Elsevier Academic Press, 2005.

[3] Malik M, Camm J. Dynamic Electrocardiography. Blackwell Future 2004

[4] Barquero-Pérez O, Goya-Esteban R, Alonso-Atienza F, et al. A review on recent patents in digital processing for cardiac electric signals (I): Advanced Systems and Applications 2009: In this issue.

[5] Simske, S.J., Blakley, D.R., Zhang, T.: US20060235322 (2006)

[6] Diziubinski, M. : WO2007043903 (2007).

[7] Xue, J.Q., Brodnick, D.E.: US20080082013 (2008)

[8] Albrecht, P., Grimshaw, P.G. Librett, K.S., Arnold, J.M.: US5908393 (1999).

[9] Kovtun, V.V., Dodson, R., Bange, J.E.: US20036512944B1 (2003).

[10] Olson, D., Schweizer, SO., Hart, T.R., Van Ess, D. US20030078510A1 (2003).

[11] Stadler, R., Shannon, N.: WO0057778 (2000).

[12] Logier, R., Dassonneville, A.: WO02069178 (2002)

[13] MacAdam, DP., Wang, P.J., Yang, S., Shah, D. : US20040127805 (2004).

[14] Yonce, D.J. : US20030055349 (2003).

[15] Korhonen, P.: WO2004089210 (2004)

[16] Danehorn, K., Harmat, P., Milton, P., Pang, L., Stridh, M., Sörnmo, L.: EP1808125A1 (2007).

[17] Buckles, D.S., Warner, A.F.: US20006027500 (2000)

[18] Ferek-Patric, B.: US20030204184 (2003).

[19] Saumarez, R.: GB2439562A (2008).

[20] Bullinga, J.R.: US20070055167A1 (2007)

[21] Narayan, S.M., Bhargava, V.: US20040059237 (2004).

[22] Brodnick, D., Hernke, D., Young, B, Albert, D., Wolfe, R., Gray, J., Schluter, P.: US20080051844 (2008).

[23] Casavant, D.A., Collins, R., Brown, B.L., Gunderson, B.D.: WO2006104849 (2006).

[24] Gunderson, B.D., Patel, A.S., Bounds, C.A., Brown, B.L.: US20060235476 (2006).

[25] Rajasekhar, S.V.: WO03022356 (2003).

[26] Zhu, Q., Gryzwa, M., Zhang, G.A.: WO0067840 (2000).

[27] Palreddy, S., Ricci, C., Lin, Y.: US20040106957 (2004).

[28] Aström, M., Sörnmo, L., Olmos, S.: WO03034917 (2003).

[29] Thomas, B., Millerhagen, J., Gebauer, M.: US20060178707 (2006).

[30] Fischell, D., Fischell, T, Harwoo, J., Fischell, R., Johnson, S, Hopenfeld, B, Sasha, J.: US20070093720 (2007).

[31] Day, M.C., Fischell, D., Schweiger, A.J.: US20080139954 (2008).

[32] Bornzin, G.A.: US2007286874 (2007).

[33] Snell, J.D., Borzin, G.A.: US20050059897 (2005).

[34] Borowitz, L.A., Combs, W.J.: US20060064027 (2006).

[35] Bardy, G.H., Rissmann, W.J., Ostroff, A.H., Erlinger, P.J., Allavatam, V.: US20030097153 (2003).

[36] Sauer, W.H., Cazares, S.M., Li, D.: US20080125824 (2008). 
[37] Kim, J., Bocek, J.M., Lovett, E.: WO2005065772 (2005).

[38] Kim, J., Bocek, J.M., Lovett, E.: US20050149125 (2005).

[39] Saba, S.: WO2004045385 (2004).

[40] Patel, A.S., Webb, J.D., Gunderson, B., Brown, B.L., Carlson, D.B.: US20070239043 (2007).

[41] Carlson, D.B.: WO03092494 (2003).

[42] Ding, J., Yu, Y., Kramer, A., Baumann, L.: US20040147966 (2004).

[43] Kramer, A.P., Kadhiresan, V., Ding, J., Baumann, L., Vanderline, S.: US20070208386 (2007).

[44] Swerdlow, C.: US20080033494 (2008).

[45] Holmstroem, N., Obel, M., Björling, A., HedBerg, S.: EP1380318A1 (2004).

[46] Elghazzawi, Z.E., Akiyama, E.N., Vega, C.D., Boucher, D.R.: US20060122648 (2006).

[47] Naragawa, M.: WO2008020369 (2008).

[48] Felblinger, J., Cansell, A., Meyer, D.: WO9210805 (1992).

[49] Brewer, J.E., Donohoo, A.N., Olson, K.F.: US20016263238 (2001).

[50] Stickney, R.E., Piraino, D.W., O'Hearn, P.J., Jayne, C.P.: US20080103402 (2008).
[51] Baura, G.D., Malecha, J.R., Bradley, P.W.: US20067149576 (2006).

[52] Marcovecchio, A.F., Geheb, F.J., Boucher, D.R.: US20050043763 (2005).

[53] Freeman, G.A., Elghazzawi, Z.E., Geheb, F.J., Parascandola, M. WO2005099818 (2005).

[54] Kontothanassis, L., Logan, B., Goddeau, D.: US20070073177 (2007).

[55] Korzinov, L.N., Le, D.V.: US20040010201 (2004)

[56] Kappenberger, L., Vesin, J.M.: WO2004047628 (2004).

[57] Xi, C.Q., Shkurovich, S.: US2007308308 (2007).

[58] Lohman, J.E., Lohamn, T.L. : US20040215091 (2004).

[59] Bashour, C.A., Gopakumaran, N., Visinescu, V., Xu, M., Li, L., Bakri, M.H.: US20080167567 (2008).

[60] Sitrick, D.H.: US4492235 (1985).

[61] Sweeney, RJ, Ricci, C.: WO2005002669 (2005).

[62] Moreno, PJ, Goddeau D, Logan BT.: US20050222508 (2005).

[63] Cohen, E.: WO2006048881 (2006). 